\title{
Loss of function of NaPiIIa causes nephrocalcinosis and possibly kidney insufficiency
}

Dinour, Dganit ; Davidovits, Miriam ; Ganon, Liat ; Ruminska, Justyna ; Forster, Ian C ; Hernando, Nati ; Eyal, Eran ; Holtzman, Eli J ; Wagner, Carsten A

\begin{abstract}
BACKGROUND Inherited metabolic disorders associated with nephrocalcinosis are rare conditions. The aim of this study was to identify the genetic cause of an Israeli-Arab boy from a consanguineous family with severe nephrocalcinosis and kidney insufficiency. METHODS Clinical and biochemical data of the proband and family members were obtained from both previous and recent medical charts. Genomic DNA was isolated from peripheral blood cells. The coding sequence and splice sites of candidate genes (CYP24A1, CYP27B1, FGF23, KLOTHO, SLC34A3 and SLC34A1) were sequenced directly. Functional studies were performed in Xenopus laevis oocytes and in transfected opossum kidney (OK) cells. RESULTS Our patient was identified as having nephrocalcinosis in utero, and at the age of 16.5 years, he had kidney insufficiency but no bone disease. Genetic analysis revealed a novel homozygous missense mutation, Arg215Gln, in SLC34A1, which encodes the renal sodium phosphate cotransporter NaPiIIa. Functional studies of the Arg215Gln mutant revealed reduced transport activity in Xenopus laevis oocytes and increased intracellular cytoplasmic accumulation in OK cells. CONCLUSIONS Our findings show that dysfunction of the human NaPiIIa causes severe renal calcification that may eventually lead to reduced kidney function, rather than complications of phosphate loss.
\end{abstract}

DOI: https://doi.org/10.1007/s00467-016-3443-0

Posted at the Zurich Open Repository and Archive, University of Zurich

ZORA URL: https://doi.org/10.5167/uzh-126327

Journal Article

Accepted Version

Originally published at:

Dinour, Dganit; Davidovits, Miriam; Ganon, Liat; Ruminska, Justyna; Forster, Ian C; Hernando, Nati; Eyal, Eran; Holtzman, Eli J; Wagner, Carsten A (2016). Loss of function of NaPiIIa causes nephrocalcinosis and possibly kidney insufficiency. Pediatric Nephrology, 31(12):2289-2297.

DOI: https://doi.org/10.1007/s00467-016-3443-0 


\section{ABSTRACT}

\section{Background}

Inherited metabolic disorders associated with medullary nephrocalcinosis are rare conditions, the most studied being Dent disease, familial hypomagnesemia with hypercalciuria and nephrocalcinosis, and renal hyperoxaluria. More recently, loss-of-function mutations of the CYP24A1 gene, encoding for the Vitamin D-24-hydroxylase, were found to cause nephrocalcinosis in both infants and adults. The aim of this study was to identify the genetic cause of an Israeli-Arab boy from a consanguineous family with severe nephrocalcinosis and kidney insufficiency.

\section{Subjects and Methods}

Clinical and biochemical data of the proband and family members were obtained both from previous medical charts and from recent evaluation. Genomic DNA was isolated from peripheral blood cells. The coding sequence and splice-sites of candidate genes (CYP24A1, CYP27B1, FGF23, KLOTHO, SLC34A3, and SLC34A1) were amplified by PCR and sequenced directly. Functional studies were performed in Xenopus laevis oocytes and in transfected opossum kidney (OK) cells.

\section{Results}

Our patient was identified with nephrocalcinosis in utero and at the age of 16.5 years had kidney insufficiency but no bone disease. Genetic analysis revealed a novel homozygous missense mutation, $\mathrm{R} 215 \mathrm{Q}$, in $S L C 34 A 1$, which encodes the renal sodium-phosphate cotransporter NaPiIIa. Functional studies of the R215Q mutant 
NaPiIIa revealed reduced transport activity in Xenopus laevis oocytes and increased intracellular cytoplasmic accumulation in opossum kidney cells.

\section{Conclusion}

Our findings show that dysfunction of the human NaPiIIa may cause severe renal calcification that eventually reduces kidney function, rather than complications of phosphate loss.

Keywords: NaPilla, nephrocalcinosis, SLC34A1 mutation, vitamin D

\section{Short Summary}

We identified a patient with nephrocalcinosis in utero due to a novel homozygous missense mutation in the renal sodium-phosphate cotransporter NaPiIIa. Our findings show that dysfunction of the human NaPiIIa may lead to severe renal calcification that eventually reduces kidney function, rather than complications of phosphate loss.

\section{INTRODUCTION}


Nephrocalcinosis, defined as renal parenchyma calcification, is caused by a number of conditions, which involve dysregulation of calcium homeostasis and may lead to acute or chronic kidney injury [1].

Inherited metabolic disorders associated with nephrocalcinosis with or without nephrolithiasis are rare conditions, the most studied are Dent disease, familial hypomagnesemia with hypercalciuria and nephrocalcinosis (FHHNC) and renal hyperoxaluria $(\mathrm{PH})$. More recently, loss-of-function mutations of the CYP24A1 gene, encoding for the Vitamin D-24-hydroxylase, were discovered to be a main cause of severe infantile hypercalcemia, hypercalciuria and nephrocalcinosis [2],[3].

NaPilla is a member of the SLC34 family of sodium-driven phosphate cotransporters, mainly expressed in the apical brush border membrane of the kidney proximal tubule [4]. Extensive studies in murine models have shown that either complete deletion of the SLC34A1 gene or homozygosity loss-offunction NaPilla mutations reduce renal reabsorption of phosphate, alter phosphate homeostasis and lead to renal calcification [5],[6].

However, until recently the clinical effects of NaPilla mutations in human were controversial. It has remained unclear whether human heterozygous mutations may be the cause of kidney stones $[7,8]$. The clinical findings in the first reported homozygous NaPilla mutation in human included Fanconi syndrome and bone disease without hypercalciuria or nephroalcinosis [9], while another paper described hypercalcemia and nephrocalcinosis without skeletal dysfunction [10]. Recent publications strengthen the involvement of SLC34A1 in hypercalcemia and kidney stones. A genome-wide-associationstudy among 5419 kidney stone cases found an association to a rare missense variant in SLC34A1 [11]. Halbritter et. al. reported that out of 272 
kidney stone patients screened for mutations in 30 genes suspected to be associated with kidney stones, one patient was found with compound heterozygous mutations in s/c34a1 [12]. Finally, Schlingmann et. al. reported causative mutations in SLC34A1 in cases of idiopathic infantile hypercalcemia [13].

We studied an Israeli-Arab adolescent from a consanguineous family with severe nephrocalcinosis and kidney failure, and identified by the candidate gene approach a homozygous NaPilla missense mutation, p.Arg215Gln, as underlying the disease. The proband's mutation is described in Schlingmann et. al. ([13], supplement material), but functional analysis was not performed. Here we show that the potential pathogenic effect of this mutation is suggested by in silico analysis and is verified by functional studies in Xenopus Laevis oocytes and in opossum kidney cells.

\section{SUBJECTS AND METHODS}

\section{$\underline{\text { Subjects }}$}

We studied an Israeli-Arab adolescent boy with nephrocalcinosis and kidney insufficiency, and several members of his extended family. Clinical data were obtained both from previous medical charts and from recent evaluation. The study protocol was approved by the institutional review board of the Chaim Sheba Medical Center, Israel. Informed consent for genetic analysis and medical evaluation was obtained from all studied subjects. Consent for the minors was given by their parents. 


\section{$\underline{\text { Candidate gene sequencing }}$}

The history of hypocalcemia and nephrocalcinosis along with increased 1,25 dihydroxyvitamin $D_{3}$ and suppressed PTH levels prompted us to evaluate genes associated with calcium and phosphate homeostasis.

Genomic DNA was isolated from peripheral blood cells applying the ArchivePure DNA Blood Kit (5 PRIME, USA) according to the manufacturer's instructions. The coding sequence and splice-sites of CYP24A1, CYP27B1, FGF23, KLOTHO, SLC34A3, and SLC34A1 were amplified by PCR using intronic primers. Primers for CYP24A1 were previously described [3]. Sequence of the other primers will be provided on demand. All PCR products were sequenced directly (ABI Prism 3100; Applied Biosystems, Foster City, CA).

\section{Preparation of Plasmid Constructs}

Full-length $h S L C 34 A 1$ subcloned either into the KSM oocyte expression vector or into pEGFP-C1 (Clontech) [14] was used as template to generate the p.Arg215Gln mutant. The mutation was introduced by site-directed mutagenesis using the Quick-Change kit (Stratagene). Mutagenic primers were designed using the Stratagene web based QuickChange® Primer Design software Program. The plasmid containing the generated mutation was isolated using QIAprep Spin Miniprep (Qiagen, 27106) and the correct mutation was confirmed by sequencing (Microsynth, Balgrist, Switzerland). One clone from each construct was used for the experiments. 


\section{$\underline{\text { Cell culture and transient transfections }}$}

Opossum kidney cells (clone 3B/2) were cultured in DMEM / Ham's F-12 medium (1:1) supplemented with $10 \%$ fetal calf serum, $2 \mathrm{mM}$ glutamine, 20 $\mathrm{mM}$ Hepes and $50 \mathrm{IU} / \mathrm{ml}$ penicillin/streptomycin as previously reported [15]. Cells were plated on coverslips in 12 multiwell plates (TPP), and cultures were transfected with either wild type (WT) hNaPilla fused to pEGFP-C1 vector or with the mutant described above. Cultures were transfected at about $70 \%$ confluency by an overnight incubation in $500 \mu \mathrm{I}$ OPTIMEM (GIBCO, 31985-047) containing $1 \mu \mathrm{g}$ of DNA and $3 \mu \mathrm{l}$ of Lipofectamine ${ }^{\mathrm{TM}} 2000$ Reagent (Invitrogen, 11668-019). We performed two independent experiments, each in duplicates or triplicates.

\section{Actin staining and confocal microscopy of OK cells}

Upon expression of clear patches of WT hNaPilla signal (two to three days after transfection), cells were fixed with paraformaldehyde and permeabilised with saponin as described previously [16]. Thereafter, actin was stained by incubation with Texas Red®-X phalloidin (Invitrogen) diluted 1:500. After incubation for $30 \mathrm{~min}$ in the dark, cells were washed three times with PBS/saponin and once with PBS. The coverslips were then mounted on microscope slides using DakoGlycergel® Mounting Medium. The subcellular final locations of the transfected cotransporters were analyzed by Confocal Laser Scanning Microscopy (Leica SP2) using a 63x oil immersion objective at the Center for Microscopy and Image Analysis at the University of Zurich.

Expression of hNaPilla and the p.Arg215Gln mutant in Xenopus Laevis oocytes 
Female X. laevis frogs were purchased from Xenopus Express (France) or African Xenopus Facility (R. South Africa). Portions of ovaries were surgically removed from frogs anesthetized in MS222 (tricainemethansulphonate) and cut in small pieces. Oocytes were treated for 45 min with collagenase (crude type 1A) $1 \mathrm{mg} / \mathrm{ml}$ in $100 \mathrm{Na}$ solution (see below; without $\mathrm{Ca}^{2+}$ ) in presence of $0.1 \mathrm{mg} / \mathrm{ml}$ trypsin inhibitor type III-O. Healthy stage $\mathrm{V}-\mathrm{VI}$ oocytes were selected, maintained at $16^{\circ} \mathrm{C}$ in modified Barth's solution (in mM: $88 \mathrm{NaCl}, 1$ $\mathrm{KCl}, 0.41 \mathrm{CaCl}_{2}, 0.82 \mathrm{MgSO}_{4}, 2.5 \mathrm{NaHCO}_{3}, 2 \mathrm{Ca}\left(\mathrm{NO}_{3}\right)_{2}, 7.5 \mathrm{HEPES}, \mathrm{pH} 7.5$ adjusted with Tris and supplemented with $5 \mathrm{mg} / \mathrm{l}$ doxycyclin and $5 \mathrm{mg} / \mathrm{l}$ gentamicin). All animal procedures were conducted in accordance with the Swiss Cantonal and Federal legislation relating to animal experimentation.

Capped cRNA was synthesized in vitro using Megascript T3 kit (Ambion) in the presence of cap analog (New England Biolabs). Oocytes were injected with $50 \mathrm{nl}$ of cRNA (200 $\mathrm{ng} / \mathrm{\mu l}$ ). Experiments were performed 3 days after injection. Non-injected control oocytes (NI), oocytes expressing hNaPilla WT and the p.Arg215Gln mutant (6-10 oocytes/group) were first allowed to equilibrate in $100 \mathrm{Na}$ solution (in mM: $100 \mathrm{NaCl}, 2 \mathrm{KCl}, 1.8 \mathrm{CaCl}_{2}, 10 \mathrm{HEPES}$, pH 7.4 adjusted with Tris) without tracer. After aspiration of this solution, oocytes were incubated in $100 \mathrm{Na}$ solution containing $1 \mathrm{mM}$ cold $\mathrm{P}_{\mathrm{i}}$ (added from a $1 \mathrm{M} \mathrm{K}_{2} \mathrm{HPO}_{4} / \mathrm{KH}_{2} \mathrm{PO}_{4}$ stock premixed to give $\mathrm{pH}$ 7.4) and ${ }^{32} \mathrm{P}_{\mathrm{i}}$ (specific activity $10 \mathrm{mCi}$ mmol-1 Pi, Perkin Elmer). Uptake proceeded for $10 \mathrm{~min}$, then oocytes were washed 4 times with ice-cold $0 \mathrm{Na}$ solution ( $\mathrm{Na}$ replaced by choline) containing $2 \mathrm{mM} \mathrm{P}$ and lysed individually in 2\% SDS for $10 \mathrm{~min}$ before addition of the scintillation cocktail. The amount of radioactivity in each oocyte was measured by scintillation counting (Tri-Carb 29000TR, Packard). 
All voltage clamp experiments were performed using a two-electrode voltage clamp (TEC-10CX, NPI, Tamm, Germany). Oocytes were impaled with microelectrodes filled with $3 \mathrm{M} \mathrm{KCl}$, with a typical resistance of $<1 \mathrm{M} \Omega$. The temperature of the recording chamber was monitored using a thermistor probe (TS-2, NPI, Tamm, Germany) placed close to the oocyte. The temperature of the recording chamber and incoming superfusate were regulated using Peltier cooling elements driven by a continuous feedback controller (TC-10, NPI Tamm, Germany). Data acquisition was performed using a 1440 Digidata (Molecular Devices Corp, USA). For recordings at constant holding potential, currents were acquired at $>20$ samples/s and filtered at $10 \mathrm{~Hz}$. Faster sampling rates (up to $20 \mathrm{k}$ samples/s) were used for voltage step recordings with filtering (digital and analog) adjusted accordingly. Steady-state currents were obtained using a protocol in which membrane voltage steps were made from the holding potential $(\mathrm{Vh})=-60 \mathrm{mV}$, to test voltages in the range -140 to $+40 \mathrm{mV}$ in $20 \mathrm{mV}$ increments. The steady-state Pi-dependent current (IPi) was obtained by subtracting control traces (in $100 \mathrm{Na}$ solution) from the corresponding traces in the presence of Pi. Data was rejected if contaminated by endogenous $\mathrm{Cl}^{-}$-currents. Experiments were performed with 0.1 and $1 \mathrm{mM}$ in the presence of $100 \mathrm{mM} \mathrm{Na}$ or in a solution where $50 \mathrm{mM} \mathrm{NaCl}$ had been replaced by $50 \mathrm{mM} \mathrm{LiCl}$.

\section{RESULTS}

Clinical characteristics of the patient and family 
A 16.5-year-old adolescent was referred to our clinic at the age of 12 years for evaluation of congenital nephrocalcinosis. He is the first child of healthy consanguineous parents of Israeli-Arab origin (fig. 1a) with other three healthy siblings. A paternal aunt and grandmother and a maternal uncle were previously diagnosed with nephrolithiasis, the aunt had undergone extracorporeal lithotripsy. No cases of end-stage renal failure in the family were reported.

The patient was born at term in another hospital. Medullary nephrocalcinosis was suspected on fetal ultrasound and confirmed on neonatal and later repeated sonographic exams.

The child developed normally and was asymptomatic, but nephrocalcinosis persisted on repeated ultrasound examinations with normal sized kidneys growing along the $30-50^{\text {th }}$ percentile for age. However, on the most recent imaging a left kidney lower pole stone was found along with cortical thinning and prominent diffuse medullary calcifications (fig. 1b).

Physical examination during the follow-up was normal, with growth and blood pressure - within normal range for age. Up to the age of 16.5 years the patient did not show any sign of rickets or any other bone disease.

Laboratory investigations in our clinic revealed normal serum calcium and phosphorus levels, slightly depressed PTH levels- 12.3 - 16.5 ng/L (normal $15-65 \mathrm{ng} / \mathrm{L})$ and elevated 1,25 dihydroxyvitamin D: $166-230 \mathrm{pmol} / \mathrm{L}$ (normal 39-160 pmol/L) with normal 25 hydroxyvitamin D levels. Urinary calcium/creatinine was within low-normal range: $0.05-0.1$ in repeated $24-$ hour urine collections. No phosphaturia was found with TRP (tubular reabsorption of phosphate) values of $92 \%-96.7 \%$ on repeat samples and 
TmP/GFR of $2.9-4.02 \mathrm{mg} / \mathrm{dl}$. Citrate excretion was slightly depressed with normalization after lemon juice supplement. Urinary oxalate, uric acid and cystine excretion were also normal. Normal urinary lysozyme concentration was found and normal albumin/creatinine ratio. GFR estimated by creatinine clearance was decreased to $70 \mathrm{ml} / \mathrm{min} / 1.73 \mathrm{~m}^{2}$.

Unfortunately, we could not retrieve detailed data on the relevant laboratory parameters before the referral to our clinic, especially for the neonatal period when, according to the parents and the family physician, he was hospitalized due to hypercalcemia and nephrocalcinosis already present. The only relevant findings were serum calcium of $12.4 \mathrm{mg} / \mathrm{dl}$ and phosphorus $3.2 \mathrm{mg} / \mathrm{dl}$ at the age of 5 months (normal range for age: calcium $8.8-10.8 \mathrm{mg} / \mathrm{dl}$, phosphorus $3.8-6.5 \mathrm{mg} / \mathrm{dl}$ ) and hypercalciuria in the range of $0.25-0.35$ calcium/creatinine found on urine samples at the ages of 6-10 years (normal range for age: calcium/creatinine $<0.2)$.

We also evaluated nine family members, including the three subjects with a history of nephrolithiasis. All of them had normal kidney function, normal serum calcium and phosphorus values and normal calcium/creatinine and phosphorus/creatinine ratio in urine (data not shown).

\section{Genetic analysis of candidate genes}

We first examined several genes that are known to be involved in the regulation of calcium and vitamin $\mathrm{D}$ handling and have been linked to nephrocalcinosis or kidney stones. We sequenced the genes CYP24A1, CYP27B1, KLOTHO and FGF23 and found no mutation. We then sequenced SLC34A1 and SLC34A3, the genes coding for the renal phosphate renal transporters NaPilla and NaPillc. In SLC34A3, in which mutations are known 
to cause hypophosphatemic rickets with hypercalciuria and hyperphosphaturia, we identified a heterozygous insertion c.967_968insAGCTCCTGGCCG - that was also present in the healthy father (data not shown).

In SLC34A1 we identified a novel homozygous missense mutation, p.Arg215Gln (fig. 1c). Several family members, with or without a history of nephrolithiasis, were found to be heterozygous carriers of this mutation. However, in the proband's grandmother, who reported having kidney stones in the past no mutation was found (fig. 1a).

\section{$\underline{\text { In Silico studies }}$}

The new mutation detected in the SLC34A1 gene (hg19:chr5:176814874) was examined in various large data sets to exclude it from being a polymorphism. A variant in this position was not evident in the 1000 genomes project [17] or in the ESP (ESP http://evs.gs.washington.edu/EVS/) project (zero alleles). In addition, a variant in this position does not appear in the Exac browser which aggregates information of more than 60,000 individuals (Exome Aggregation Consortium - ExAC, Cambridge, MA, http://exac.broadinstitute.org) either. Finally, we examined 100 normal Israeli-Arab genomes, and did not find even a single mutated allele.

Furthermore, the mutation we describe replaces an amino acid highly conserved among the members of the SLC34 family (fig. 2a). 
The mutation is located in the last nucleotide of the sixth exon. As such, it might affect the splicing donor motif. cDNA analysis of the proband and his father showed that this is not the case (data not shown).

No experimental structure has been solved for this protein, so a careful structural insight regarding this mutation is missing. No structure for a close homolog is available so it is also difficult to construct a confident model. However, Fenollar-Ferrer et al. [18] constructed a model based on the structure of the Vibrio cholerae $\mathrm{Na}+$ dicarboxylate transporter, which shares overall sequence identity of around $15 \%$ with human SLC34A1. They suggest inverted structural repeats in the overall topology, like often found in other families of transporters. The model suggests that the mutation is located in the first structural repeat unit, in the $\mathrm{N}$-terminal domain, in or close to the cytoplasmic side of the membrane (fig. 2b).

\section{Functional studies}

\section{Transport activity of mutant NaPilla}

Expression of human wild type NaPilla in Xenopus laevis oocytes induced sodium-dependent phosphate uptake whereas oocytes not injected with cRNA showed only negligible phosphate uptake. Moreover, the R215Q NaPilla transporter mediated some phosphate transport, significantly higher than in non-injected oocytes but also significantly less than in wild type expressing oocytes (Figure 3a). 


\section{Electrophysiological characterization of mutant NaPilla}

We assessed the electrogenic behavior of the R215Q mutant by functional expression in Xenopus oocytes using the standard two electrode voltage clamp (TEVC) technique. We used our standard screening assay [19] to compare the normalized response to $0.1 \mathrm{mM} \mathrm{Pi}$ (in $100 \mathrm{Na}$ ); $50 \mathrm{mM} \mathrm{Na}(1 \mathrm{mM}$ Pi) and $50 \mathrm{mM} \mathrm{Na}+50 \mathrm{MM} \mathrm{Li} \mathrm{(1} \mathrm{mM} \mathrm{Pi}$ (Fig 3b). Compared with the WT hNaPi-Ila (fig. 3b left panel), the mutant showed a more curvilinear I-V in response to $1 \mathrm{mM} \mathrm{Pi}$, suggesting that the removal of charge at 215 had affected the voltage dependence (Fig $3 \mathrm{~b}$ right panel). The response to 0.1 $\mathrm{mM}_{\mathrm{Pi}}(100 \mathrm{Na}+0.1 \mathrm{Pi})$ was suppressed compared with the WT suggesting that the mutagenesis had marginally compromised the apparent affinity for $P_{i}$ $\left(K_{0.5^{P i}}\right)$ Based on this two state assay, we predict that this would amount to an approximately 2 -fold increase in $K_{0.5}{ }^{\mathrm{Pi}}$ (see supplementary figure, Fennolar [19]). Moreover, whereas for the WT, the normalized responses to a $50 \%$ reduction in external $\mathrm{Na}^{+}(50 \mathrm{Na}+1 \mathrm{Pi})$ or replacement of $50 \%$ external $\mathrm{Na}^{+}$ ions with $\mathrm{Li}^{+}$ions $(50 \mathrm{Na} 50 \mathrm{Li}+1 \mathrm{Pi})$ were similar, $\mathrm{R} 215 \mathrm{Q}$ showed a slightly increased response with respect to the $\mathrm{Li}+$ substitution.

\section{Expression in renal proximal tubule cells}

EGFP-tagged wild type NaPilla was transiently transfected into the renal opossum kidney cell line, a model for the proximal tubule. Wild type NaPilla localized to the luminal brush border membrane as indicated by the yellow color resulting from colocalization with actin (stained in red) expressed in apical microvilli (fig. 3c left panel). Lateral views of the same cell indicate that a substantial part of NaPilla is found in the apical membrane as suggested by 
only faint green intracellular staining. The R215Q mutant localized also in part to the brush border membrane and colocalized there with actin (yellow overlay). However, in contrast to the wild type situation, distinct intracellular green staining was observed suggesting partial retention of the mutant protein (3c right panel).

\section{DISCUSSION}

We report that a homozygous missense mutation in the sodium-phosphate co-transporter NaPilla, p.Arg215Gln, is the likely cause of nephrocalcinosis and kidney insufficiency in our patient. The causative role of this mutation in the pathogenesis of our patient's kidney disease is supported by the following findings: 1. among the 9 family members studied only the homozygous proband had nephrocalcinosis and kidney insufficiency. 2. The nucleotide variant that we identified was not found in the general population, and was absent from 200 alleles of healthy unrelated Israeli-Arab individuals. 3. The mutated amino acid, the arginine in position 215 , is highly conserved through evolution and in the SLC34 family of phosphate transporters (fig. 2a). 4. In silico studies suggest that this mutation results in a significant change of both the size and the charge in this position, and is deleterious to the protein. 5 . The results of In Vitro expression studies indicate that the single amino acid change found in our patient suffices to reduce phosphate transport and localization in the apical membrane.

Our findings are in agreement with those found in animal models of reduced or absent NaPilla function. In mice, a homozygous single amino acid substitution in NaPilla altered phosphate $(\mathrm{Pi})$ homeostasis and led to renal 
calcification [5]. NaPilla knock-out mice show increased urinary Pi excretion, hypophosphatemia, elevated serum 1,25 dihydroxyvitamin D levels, decreased serum PTH levels, hypercalcemia and hypercalciuria [6].

In humans, however, the impact of heterozygous NaPilla mutations in the formation of kidney stones is controversial and the clinical effects of homozygous mutations vary. The first study on the role of NaPilla in human kidney disease and bone demineralization was published by Prie et al in the NEJM, 2002 [7]. In this report two out of 20 patients with urolithiasis, bone deformation and hypophosphatemia were found to be heterozygous carriers of NaPilla missense mutations (A48F; V147M). Functional analysis in Xenopus Laevis oocytes demonstrated altered function and dominant negative effects of the mutated transporter. These findings were later challenged by two other studies: Virkki et al examined the function of the same two mutations and found similar apical expression of mutated and WT proteins in OK cells. Furthermore, oocyte studies excluded a dominant negative effect of both mutations [14]. Lapointe et al examined several NaPilla variants found in a cohort of 98 pedigrees with a history of multiple calcium kidney stones including non-synonymous mutations and a truncation deletion. No difference was found between WT and heterozygous carriers in terms of calcium and phosphorus excretion, so the authors concluded that the variants described had no clinical significance [8].

Interestingly, several reports described either hypophosphatemia [20] or infantile hypercalcemia and nephrocalcinosis [21] in patients with Sotos syndrome, caused by a heterozygous microdeletion on chromosome $5 \mathrm{q} 35$, which removes both the NSD1 and NaPilla genes. Sotos syndrome is characterized by bone overgrowth and mental retardation due to 
haploinsufficiency of the NSD1 gene. The calcium and phosphorus disorders were assumed to be the consequence of NaPilla haploinsufficiency, but it is not clear whether other changes due to the deletion also contribute to the phenotype.

The first report of a homozygous NaPilla mutation in human described two Israeli-Arab siblings with hypophosphatemic rickets, hypercalciuria, highly elevated 1,25 dihydroxyvitamin $D$ levels and partial Fanconi syndrome. Both carried an in-frame duplication of 21 bp that was shown in Xenopus Laevis oocytes and opossum kidney cells to prevent transport of the protein to the cell membrane, thereby leading to complete loss-of function of the transporter [9]. The occurrence of Fanconi syndrome in the context of SLC34A1 mutation is not clear. In two different mouse models of SLC34A1 deficiency due to genetic deletion or compound heterozygous mutations no signs of Fanconi syndrome have been observed [22]. Suggested explanations include a specific toxic tubular effect of this particular mutation due to intracellular accumulation or an additional mutation in this consanguineous family [23].

Rajagopal et al reported another mutation in SLC34A1, p.Arg495His, in two siblings who presented with hypercalciuria and nephrocalcinosis [10]. Interestingly, according to our model, the p.Arg495His mutation was predicted to fall in a similar context as our mutation, within a cytoplasmic loop. This mutation is located in a less quality region of our model so we cannot verify/contradict their conclusion.

Screening a cohort of patients with kidney stones, Halbritter et al identified one patient compound heterozygous for the p.Val91_Ala97del and p.Arg512Cys mutations. Of note, the p.Val91_Ala97del had been identified 
previously by Lapointe et al [8], and suggested to be non-pathogenic whereas no information is available on the functional consequences of the p.Arg512Cys mutation [12].

Most recently, Schlingmann et.al. reported on 16 cases of infantile idiopathic hypercalcemia with causative autosomal recessive mutations in slc34a1. Functional studies of some of the mutations demonstrated disturbed trafficking to the plasma membrane as well as loss of phosphate transport activity. The mutation that we evaluated, p.Arg215Gln, is predicted to be harmful to the protein by most predictive tools. Since the mutation affects a charged residue and might be located in a predicted transmembrane domain, it could be expected to alter helical packing and/or mobility of the transporter in the membrane. Our in vitro results suggest indeed reduced transport function of the p.Arg215Gln mutation in the oocyte assay and impaired trafficking of the mutant protein to the plasma membrane in OK cells. This concurs with the functional results of Schlingmann et.al., although the p.Arg215Gln showed only partial reduction in phosphate transport, as opposed to the loss of phosphate transport in other mutants shown by Schlingmann et.al.

The results of the electrophysiological characterization suggest that the $\mathrm{R} \rightarrow$ Q substitution at position 215 had indeed altered the voltage-dependent kinetics and the interaction of cations with the transporter. This in turn would lead to the observed reduced apparent affinity for $\mathrm{P}_{\mathrm{i}}$. Given that under physiological conditions, the renal $\mathrm{P}_{\mathrm{i}}$ at $\mathrm{pH} 7.4$ is far in excess of $K_{0.5}{ }^{\mathrm{Pi}}$, we would conclude that the mutation had marginal effects on the transport behavior in vivo. However, we cannot exclude the possibility of a somewhat compromised contribution of NaPilla to overall proximal tubular Pi influx at low 
$\mathrm{P}_{\mathrm{i}}$ below or close to the reported $\mathrm{WT} \mathrm{K}_{0.5}{ }^{\mathrm{Pi}}$ for human NaPilla of $\sim 100 \mu \mathrm{M}$. The reduced apical expression may account for most of the observed phenotype in vivo reducing the overall capacity of the kidney to conserve phosphate.

Based on animal data, mutations in s/c34a1 leading to complete loss of function or gene deletion would be expected to cause renal phosphate loss. However, the mutation described here may exert only a mild impairment of renal phosphate reabsorption. Moreover, the increase in 1,25 dihydroxyvitamin D may fully compensate for the defect in NaPilla by upregulating NaPillc, at the expense of increased intestinal calcium absorption and subsequent renal hypercalciuria, facilitating nephrocalcinosis. Alternatively, the contribution of NaPilla to renal phosphate reabsorption may decrease by age in contrast to the situation in mice.

Unlike the phenotype in humans with mutation of the second kidney phosphate transporter, NaPillc (SLC34A3), hypophosphatemic rickets is not a frequent feature in patients with SLC34A1 mutations. Whether heterozygous NaPilla mutations can cause kidney stones remains to be proven. Our family included 6 heterozygous carriers of the mutation of which only 2 had a history of kidney stones. The proband's grandmother reported kidney stones although she carried two wild type SLC34A1 alleles. Thus, other hereditary or environmental factors contributed to the development of kidney stones in this family, and maybe also in previously reported heterozygous carriers of different SLC34A1 variants [7],[8]. Kidney stone formation on the background of heterozygous SLC34A1 mutations may provide an example of geneenvironment interactions and remain to be deciphered in more detail. 


\section{Conclusion}

We present severe nephrocalcinosis and renal insufficiency in a patient carrying a homozygous (p.Arg215Gln) mutation of the renal phosphate transporter NaPilla causing partial loss of function.

The prominent effect of this mutation is increased serum calcium and renal calcification, rather than complications of phosphate loss. Our interpretation for these findings is that the mutation described here may exert only a mild impairment of renal phosphate reabsorption, probably compensated by upregulation of NaPillc due to increased 1,25 dihydroxyvitamin D. The elevation of 1,25 dihydroxyvitamin $D$ results in increased intestinal calcium absorption, which leads to the severe nephrocalcinosis seen in our patient. Thus, mutations in SLC34A1 may increase the risk for hypercalciuria and nephrocalcinosis, eventually leading to renal insufficiency, and must be considered in young patients with such symptoms. 


\section{REFERENCES}

1. Edvardsson, V.O., D.S. Goldfarb, J.C. Lieske, et al. Hereditary causes of kidney stones and chronic kidney disease. Pediatr Nephrol, 2013. 28:1923-1942.

2. Schlingmann, K.P., M. Kaufmann, S. Weber, et al. Mutations in CYP24A1 and idiopathic infantile hypercalcemia. N Engl J Med, 2011. 365:410-421.

3. Dinour, D., M. Davidovits, S. Aviner, et al. Maternal and infantile hypercalcemia caused by vitamin-D-hydroxylase mutations and vitamin D intake. Pediatr Nephrol, 2014. 30:145-152.

4. Wagner, C.A., N. Hernando, I.C. Forster, and J. Biber. The SLC34 family of sodiumdependent phosphate transporters. Pflugers Arch, 2014. 466:139-153.

5. Iwaki, T., M.J. Sandoval-Cooper, H.S. Tenenhouse, and F.J. Castellino. A missense mutation in the sodium phosphate co-transporter Slc34a1 impairs phosphate homeostasis. J Am Soc Nephrol, 2008. 19:1753-1762.

6. Beck, L., A.C. Karaplis, N. Amizuka, A.S. Hewson, H. Ozawa, and H.S. Tenenhouse. Targeted inactivation of Npt2 in mice leads to severe renal phosphate wasting, hypercalciuria, and skeletal abnormalities. Proc Natl Acad Sci U S A, 1998. 95:53725377.

7. Prie, D., V. Huart, N. Bakouh, et al. Nephrolithiasis and osteoporosis associated with hypophosphatemia caused by mutations in the type $2 a$ sodium-phosphate cotransporter. N Engl J Med, 2002. 347:983-991.

8. Lapointe, J.Y., J. Tessier, Y. Paquette, et al. NPT2a gene variation in calcium nephrolithiasis with renal phosphate leak. Kidney Int, 2006. 69:2261-2267.

9. Magen, D., L. Berger, M.J. Coady, et al. A loss-of-function mutation in NaPi-lla and renal Fanconi's syndrome. N Engl J Med, 2010. 362:1102-1109.

10. Rajagopal, A., D. Braslavsky, J.T. Lu, et al. Exome sequencing identifies a novel homozygous mutation in the phosphate transporter SLC34A1 in hypophosphatemia and nephrocalcinosis. J Clin Endocrinol Metab, 2014. 99:E2451-2456.

11. Oddsson, A., P. Sulem, H. Helgason, et al. Common and rare variants associated with kidney stones and biochemical traits. Nat Commun, 2015. 6:7975.

12. Halbritter, J., M. Baum, A.M. Hynes, et al. Fourteen monogenic genes account for 15\% of nephrolithiasis/nephrocalcinosis. J Am Soc Nephrol, 2015. 26:543-551.

13. Schlingmann, K.P., J. Ruminska, M. Kaufmann, et al. Autosomal-Recessive Mutations in SLC34A1 Encoding Sodium-Phosphate Cotransporter 2A Cause Idiopathic Infantile Hypercalcemia. J Am Soc Nephrol, 2015. 27:604-614.

14. Virkki, L.V., I.C. Forster, N. Hernando, J. Biber, and H. Murer. Functional characterization of two naturally occurring mutations in the human sodiumphosphate cotransporter type lla. J Bone Miner Res, 2003. 18:2135-2141.

15. Reshkin, S.J., J. Forgo, and H. Murer. Functional asymmetry of phosphate transport and its regulation in opossum kidney cells: phosphate transport. Pflugers Arch, 1990. 416:554-560.

16. Pfister, M.F., E. Lederer, J. Forgo, et al. Parathyroid hormone-dependent degradation of type II Na+/Pi cotransporters. J Biol Chem, 1997. 272:20125-20130.

17. Abecasis, G.R., A. Auton, L.D. Brooks, et al. An integrated map of genetic variation from 1,092 human genomes. Nature, 2012. 491:56-65. 
18. Fenollar-Ferrer, C., M. Patti, T. Knopfel, A. Werner, I.C. Forster, and L.R. Forrest. Structural fold and binding sites of the human $\mathrm{Na}(+)$-phosphate cotransporter $\mathrm{NaPi}$ II. Biophys J, 2014. 106:1268-1279.

19. Fenollar-Ferrer, C., I.C. Forster, M. Patti, T. Knoepfel, A. Werner, and L.R. Forrest. Identification of the first sodium binding site of the phosphate cotransporter NaPi-Ila (SLC34A1). Biophys J, 2015. 108:2465-2480.

20. Levtchenko, E., J. Schoeber, and J. Jaeken. Genetic disorders of renal phosphate transport. N Engl J Med, 2010. 363:1774; author reply 1774-1775.

21. Kenny, J., M.M. Lees, S. Drury, et al. Sotos syndrome, infantile hypercalcemia, and nephrocalcinosis: a contiguous gene syndrome. Pediatr Nephrol, 2011. 26:13311334.

22. Myakala, K., S. Motta, H. Murer, et al. Renal-specific and inducible depletion of NaPiIIc/Slc34a3, the cotransporter mutated in $\mathrm{HHRH}$, does not affect phosphate or calcium homeostasis in mice. Am J Physiol Renal Physiol, 2014. 306:F833-843.

23. Friedlander, G. and D. Prie. Genetic disorders of renal phosphate transport-author reply. N Engl J Med, 2010. 363:1774-1775.

\section{Acknowledgements}

We thank the patient and his family. This study was supported in part by the Swiss National Science Foundation through the National Center for Competence in Research NCCR Kidney.CH and by the EU FP7 E-rare project "Idiopathic Infantile Hypercalcemia" to C. A. Wagner. 


\section{Figure legends}

Figure 1

A. Family tree: The arrow points to the proband. White icons depict subjects not evaluated. Grey and black are the WT and mutant allele respectively. ${ }^{*}$ indicate patients with a history of nephrolithiasis.

B. A sonogram of the probands kidney at the age of 15 years.

C. Sequence chromatogram showing WT, homozygote and heterozygote subjects for the p.Arg215Gln mutation.

Figure 2

Analysis of the conservation and structural context of the R215 residue.

A. R215 is located at a conserved site within a conserved domain.

B. The predicted location of R215 residue according to Enollar-Ferrer model is shown [15].

C. Secondary structure and accessibility predictions for SLC34A1. The mutation is predicted to fall on the border between membrane helix and cytoplasmic loop and on the border between a buried part of the protein and an exposed part.

Figure 3

A. Phosphate transport activity of wild-type and mutant p.Arg215GlnhNaPilla. ${ }^{32}$ Phosphate uptakes were performed in Xenopus oocytes 3 days after injection of cRNA encoding hNaPilla. Data are from two independent batches of oocytes, each 8-10 oocytes; NI, non injected; 
wt, wild-type. All data were normalized to the transport activity of wildtype hNaPilla.

B. Normalised electrogenic behavior of oocytes expressing the human NaPi-lla WT and mutant R215Q. Data were normalized to the response when superfusing with $100 \mathrm{Na}+1 \mathrm{Pi}$ at $-100 \mathrm{mV}$. Data from WT were modified and redrawn from Fenollar et al 2015. Each data point for $\mathrm{R} 215 \mathrm{Q}$ is mean \pm sem of $4-5$ cells.

C. Expression of EGFP-tagged human NaPilla cotransporter in renal OK cells. Cells were transiently transfected with pEGFP plasmids containing either human wild-type or mutant $\mathrm{hNaPilla}$, as well as with the empty pEGFP plasmid. Confluent cultures were viewed on a confocal microscope. Top panels - Focal planes of apical projections. Bottom panels - Cross sections. NaPilla signal is shown in green, the actin staining in red, yellow indicates colocalization of NaPilla and actin in apical microvilli. 
A.

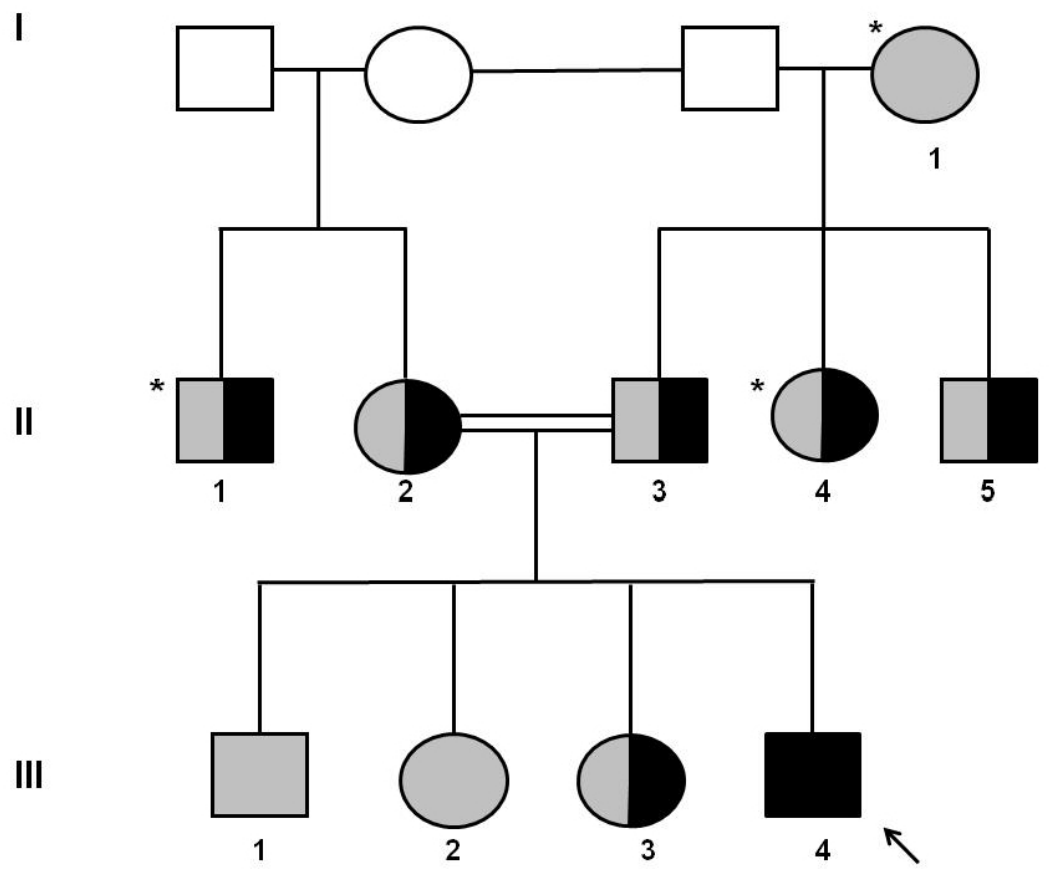

B.

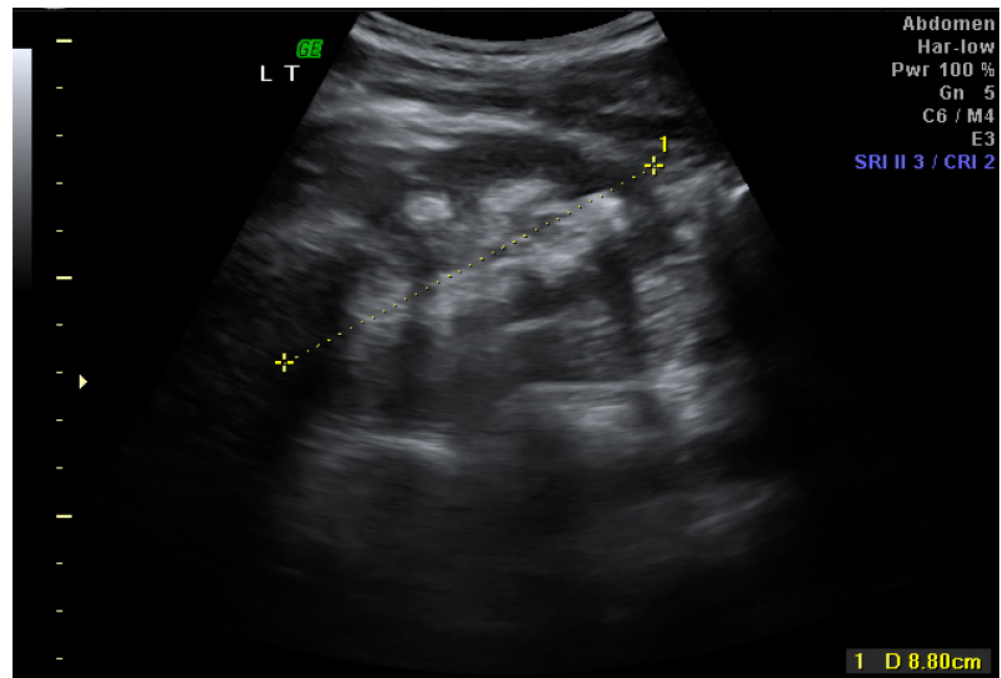

C.
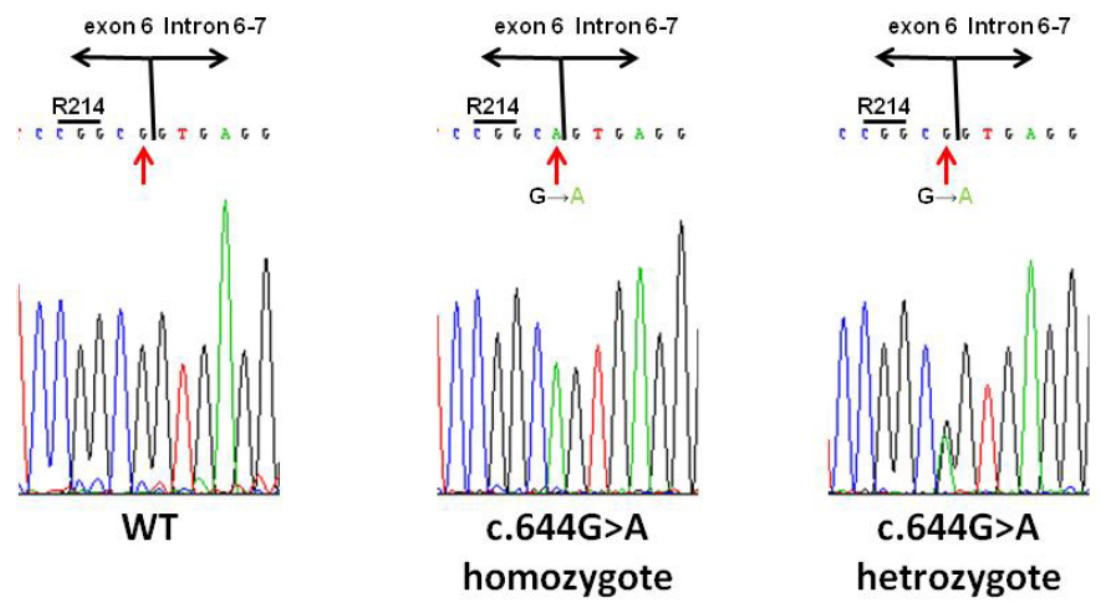
homozygote 
A.

\section{5}

SLC34A2_HUMAN/2-639 SIVT IT I VA LMQ'A GDR T'DFF R F A GA T'VHDCFNWL SV SLC34A1_RABIT/1-642 SVTNT I VALMQAGDRTDFFRAFA GATVHDCFNWL SV SLC34ALRAT/2-637 SVTNT I VALMQAGDRTDFFRAFA GATVHDCFNWLSV SLC 3441 SHEEP/1-639 SVTNT I VALMQAGDRTDFER AFAGATVHDCFNWL S V SLC34A1_MOUSE/2-637 SVTNT I VALMQAGDRTDFFR AFA GATVHDCFNWLSV SLC34A2_RAT/1-695 SI TNT I VALMQAGDRNEFFRAFAGATVHDFFNWLSV SLC34A2_MOUSE/1-697 S I TNT I VALMQA GDRNE F F R A F GA TVHDF F NWL SV SLC34A2_BOVIN/1-693 SI TNT I VALMQAGDRKEFFRAFAGATVHDFFNWLSV SLC34A2_HUMAN/2-690 S I T NT I VA LMQV GDR S E FFR AF A G A T VHDFFNWLSV SLC34A2_PONAB/1-689 S I TNT I VALMQVGDR SEFRR AFAGATVHDFFNWLSV SLC34A3_MOUSE/2-601 S I T ST LV SMAQS GDRDEF R AF SG SAVHG I FNWL TV SLC34A3_HUMAN/1-599 S I T STLVSMAQSGDRDEF CRAF SGSAVHG I FNWL T SLC34A3_RAT/2-601 SI T STLVISMAQSGDRDEFARAFGGSAVHG I FNWL TV conservation LOGO
B.

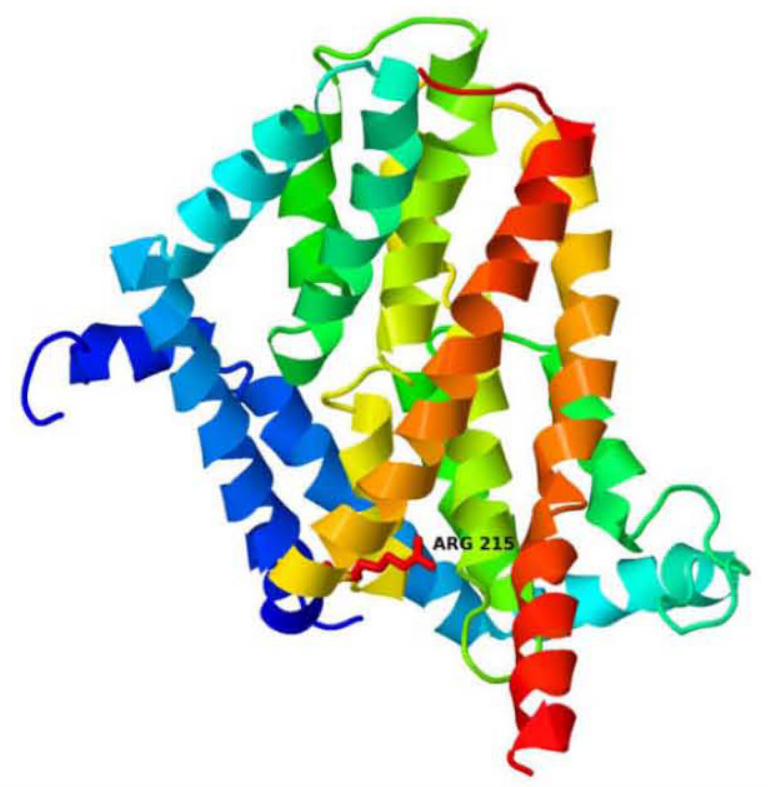

C.

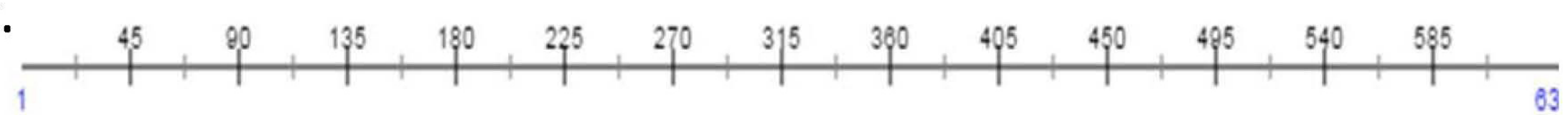

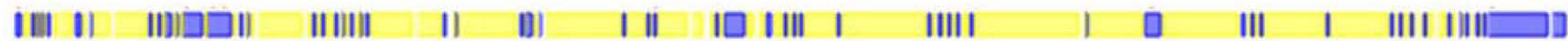
个

$\mathrm{R} 215 \mathrm{Q}$

Legend: $\square$ exposed

$\mathrm{R} 495 \mathrm{H}$

$\square$ buried

$\square$ Trans membrane helix 
A.

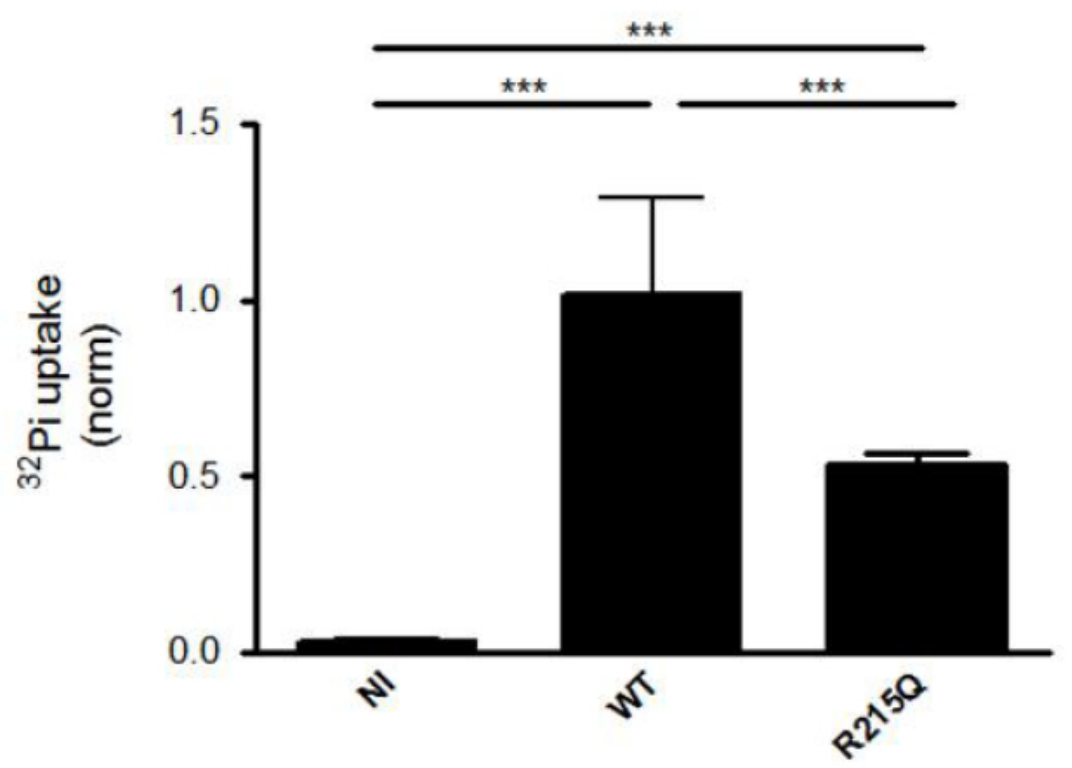

B.
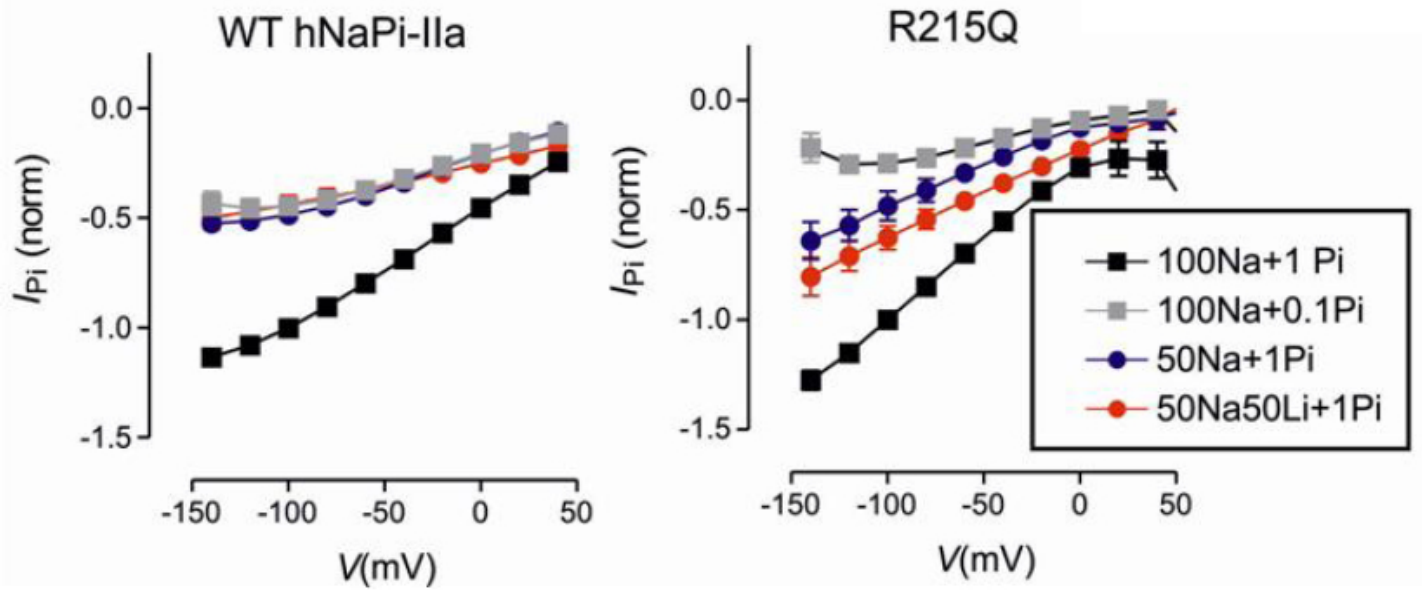

c.

hSLC34A1 WT
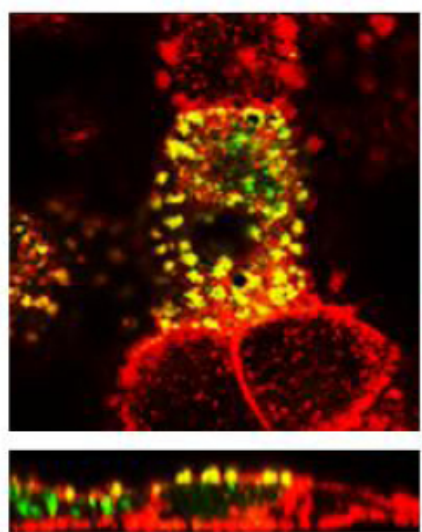

hSLC34A1 R215Q
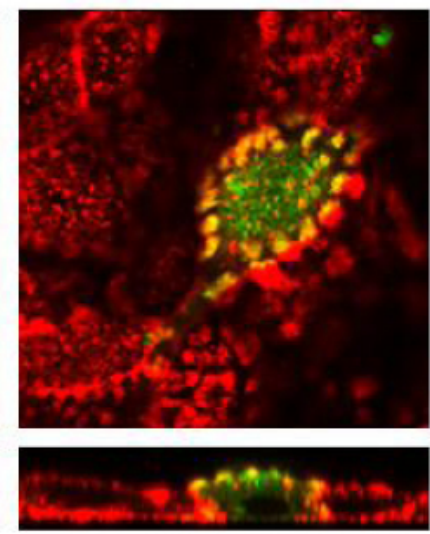